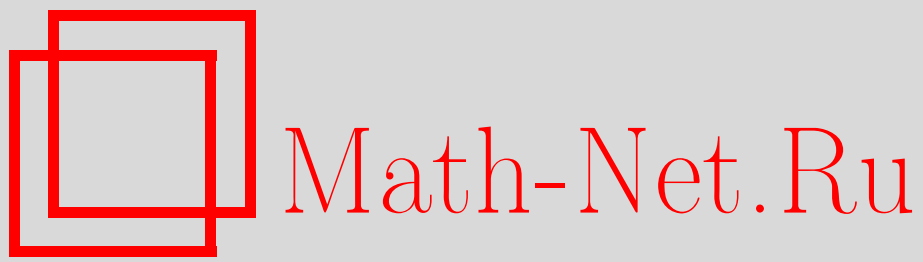

Е. Смирнов, Числовые фризы, Квант, 2020, номер 5, 15-24

DOI: https://doi.org/10.4213/kvant20200503

Использование Общероссийского математического портала Math-Net.Ru подразумевает, что вы прочитали и согласны с пользовательским соглашением http: //www.mathnet.ru/rus/agreement

Параметры загрузки:

IP : 52.6 .47 .48

26 апреля 2023 г., 17:33:06

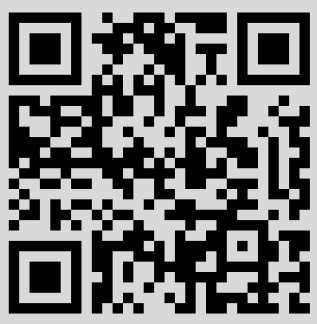




\section{Числовые фризы}

\section{E. СМИРНОВ}

Фayct:

Так пентаграмма этому виной?

Но как же, бес, пробрался ты за мной?

Каким путем впросак попался?

Мефистофель:

Изволили ее вы плохо начертить,

И промежуток в уголку остался,

Там, у дверей, - и я свободно мог вскочить.

Фауст:

Да, случай над тобой удачно посмеялся.

Так ты мой пленник, стало быть?

Вот удалось негаданно-нежданно!

И.-В.Гете. Фауст (пер. Н.Холодковского)

\section{Введение}

Фризами называются таблицы, заполненные положительными числами в соответствии с некоторым мультипликативным правилом. Такие таблицы были впервые определены в 1970-х годах в работах Дональда Кокстера и Джона Конвея. Как и многое другое, что делали эти замечательные математики, фризы можно рассматривать как некоторую математическую забаву; она состоит в том, чтобы выписывать по некоторым правилам числа и смотреть на получающиеся закономерности.

Однако оказывается, что, несмотря на простоту их определения, фризы обладают рядом весьма неожиданных свойств. Они связаны со множеством других математических понятий, в числе которых триангуляции многоугольников, числа Каталана и Фибоначчи, цепные дроби, ряды Фарея... Кроме того, оказалось, что фризы (так, конечно, тогда еще не называвшиеся) неявным образом возникали еще в работах К.-Ф.Гаусса и даже Дж.Непера - т.е. более 400 лет назад!

В XXI веке интерес к фризам среди математиков существенно вырос: выясни-

DOI: https://doi.org/10.4213/kvant20200503 лось, что фризы возникают в теории кластерных алгебр, в связи с представлениями колчанов, многообразиями Грассмана, эллиптическими функциями, обобщенными ассоциаэдрами и многими другими сюжетами из современной «серьезной» математики.

Первая русскоязычная публикация о фризах появилась в «Кванте» почти тридцать лет назад, в №3 за 1991 год - причем написана она была самим Конвеем. Этот двухстраничный текст содержал множество вопросов, над ответами на которые предлагалось подумать читателю. В послесловии от редакции было сказано: «Мы надеемся получить от вас письма с ответами на вопросы, поставленные Конвеем, и тогда вновь вернуться к числовым фризам». На часть вопросов Конвея к читателям «Кванта» мы отвечаем в этой статье.

11 апреля 2020 года Джон Хортон Конвей, один из самых удивительных и неординарных математиков нашего времени, скончался на 83-м году жизни. Посвящаю этот текст его памяти.

\section{Фризы малых порядков}

Определение и первые свойства. Начнем с определения.

Определение 1. Назовем фризом порядка $n$ таблицу $\left(t_{i j}\right)$, состоящую из $n-1$ бесконечной влево и вправо строки положительных чисел, расположенных в шахматном порядке, которая удовлетворяет следующим свойствам:

- первая и последняя строки состоят из единиц: $t_{1 j}=t_{n-1, j}=1$;

• для любых четырех чисел $a \quad d$ в c вершинах единичного ромба верно равенство $a d-b c=1$; это свойство будем называть унимодулярностью. 
ется периодичным с периодом 5, и он вновь обладает скользящей симметрией.

\section{Упражнения}

1. Перечислите все целочисленные фризы порядка 5. Сколько их?

2. Рассмотрим фриз порядка 5, у которого $x=y=\tau$. Покажите, что $\tau=\frac{1+\sqrt{5}}{2}$, и найдите остальные элементы этого фриза.

Фриз из упражнения 2 получается путем применения к правильному пятиугольнику теоремы Птолемея. Эта теорема из школьного курса планиметрии гласит, что для вписанного четырехугольника $A B C D$ верно равенство $A B \cdot C D+A D \cdot B C=$ $=A C \cdot B D$. Действительно, рассмотрим правильный пятиугольник, все стороны которого равны 1 (рис.7). Пусть его диагональ равна $\tau$. Запишем теорему Птолемея для равнобокой трапеции $A B C D$; в

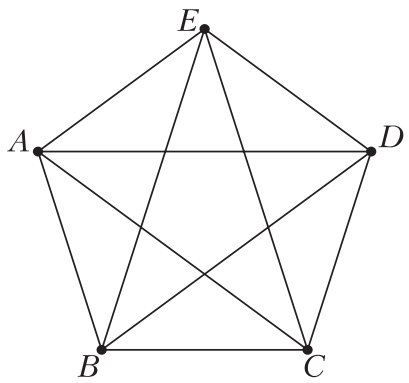
Pис. 7

этой трапеции $A B=B C=C D=1, A D=$ $=A C=B D=\tau$. Мы получим уравнение

$$
1 \cdot \tau+1 \cdot 1=\tau \cdot \tau, \text { т.е. } \tau^{2}-\tau-1=0 .
$$

Положительный корень этого уравнения как раз равен $\frac{1+\sqrt{5}}{2}$; это знаменитое золотое сечение.

Упражнение 3. Рассмотрим правильный $n$-угольник со стороной 1 и многократно применим к нему теорему Птолемея. Какой фриз порядка $n$ мы получим? Чему равны элементы в его $k$-й строке?

Pentagramma mirificum. Впервые фризы порядка 5 появились в связи со сферической тригонометрией в работах Натаниэла Тарпорли ${ }^{1}$ и Джона Непера ${ }^{2}$. Даль-

1 Nathaniel Tarporley (Torporley) (15641632) - английский математик, астроном и астролог. Некоторое время работал во Франции секретарем Франсуа Виета.

2 John Napier (1550-1617) - шотландский математик. Известен как изобретатель логарифмов и десятичной точки.
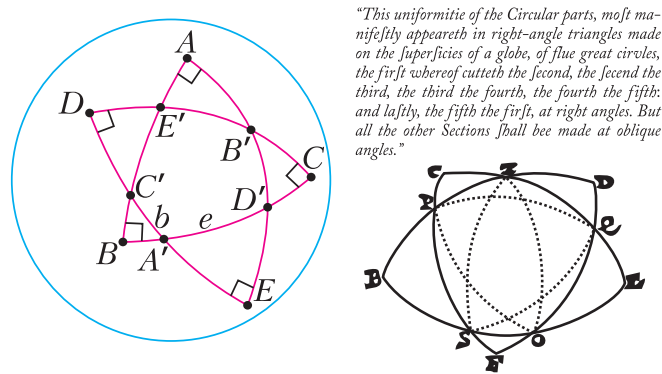

Рис. 8. Pentagramma mirificum. Справа рисунок из работы Непера (1614 г.)

нейшее развитие они получили в работах Карла-Фридриха Гаусса. Их появление было связано со следующей задачей сферической геометрии.

Рассмотрим пятиконечную звезду на сфере, все углы которой являются прямыми (рис.8). В частности, это значит, что точка $A^{\prime}$ является полюсом для большого круга $C D, B^{\prime}-$ для $D E$ и т.д.

Обозначим через $\alpha, \ldots, \varepsilon$ квадраты тангенсов длин сторон пятиугольника:

$$
\begin{gathered}
\alpha=\operatorname{tg}^{2} B^{\prime} E^{\prime}, \beta=\operatorname{tg}^{2} E^{\prime} C^{\prime}, \gamma=\operatorname{tg}^{2} A^{\prime} C^{\prime}, \\
\delta=\operatorname{tg}^{2} A^{\prime} D^{\prime}, \varepsilon=\operatorname{tg}^{2} D^{\prime} B^{\prime} .
\end{gathered}
$$

Оказывается, что эти пять величин связаны следующими соотношениями, позволяющими получить по любым двум из них остальные три:

$$
\begin{gathered}
1+\alpha=\gamma \delta ; 1+\beta=\delta \varepsilon ; 1+\gamma=\varepsilon \alpha \\
1+\delta=\alpha \beta ; 1+\varepsilon=\beta \gamma .
\end{gathered}
$$

\begin{tabular}{|c|c|c|c|c|c|c|}
\hline 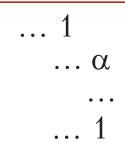 & ${ }_{\delta}{ }_{1}^{\beta}{ }^{1}$ & ${ }_{\varepsilon}^{1} \begin{array}{l}\gamma \\
1\end{array}$ & 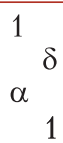 & ${ }_{\beta}^{1}$ & 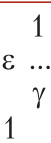 & 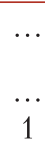 \\
\hline
\end{tabular}

Таким образом, эти величины являются элементами следующего фриза (рис.9) порядка 5.

Pис. 9

Упражнение 4. Используя соотношения (*), докажите следующее равенство (также принадлежащее Гауссу):

$$
\begin{aligned}
& \alpha \beta \gamma \delta \varepsilon=3+\alpha+\beta+\gamma+\delta+\varepsilon= \\
& =\sqrt{(1+\alpha)(1+\beta)(1+\gamma)(1+\delta)(1+\varepsilon)} .
\end{aligned}
$$

Это равенство в работе Гаусса названо schöne Gleichung, т.е. «красивым тождеством». 


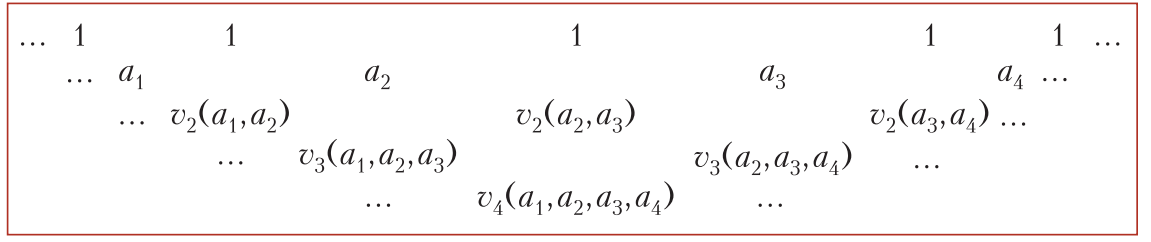

Рис. 10

\section{Континуанты}

Рекуррентное соотношение. Теперь перейдем к фризам произвольного порядка. Наша ближайшая цель - получить выражение для элементов третьей и последующих строк фриза через элементы второй строки.

Пусть дан фриз со второй строкой $\ldots, a_{1}, a_{2}, a_{3}, \ldots$ Ясно, что каждый из элементов третьей строки выражается через два элемента второй строки, стоящие непосредственно над ним (слева и справа). Далее, каждый элемент четвертой строки можно найти по правилу унимодулярности уже через три стоящих над ним элемента второй строки и так далее. Обозначим элементы третьей, четвертой и т.д. строк через $v_{2}\left(a_{i}, a_{i+1}\right), v_{3}\left(a_{i}, a_{i+1}, a_{i+2}\right)$ и т.д., как показано на рисунке 10 .

Нетрудно найти выражение для элементов третьей строки:

$$
v_{2}\left(a_{1}, a_{2}\right)=a_{1} a_{2}-1, v_{2}\left(a_{2}, a_{3}\right)=a_{2} a_{3}-1,
$$

а из них уже выразить элементы четвертой:

$$
\begin{array}{r}
v_{3}\left(a_{1}, a_{2}, a_{3}\right)=\left(v_{2}\left(a_{1}, a_{2}\right) v_{2}\left(a_{2}, a_{3}\right)-1\right) / a_{2}= \\
=a_{1} a_{2} a_{3}-a_{1}-a_{3},
\end{array}
$$

пятой:

$$
\begin{aligned}
& v_{4}\left(a_{1}, a_{2}, a_{3}, a_{4}\right)= \\
& =\left(v_{3}\left(a_{1}, a_{2}, a_{3}\right) v_{3}\left(a_{2}, a_{3}, a_{4}\right)-1\right) / v_{2}\left(a_{2}, a_{3}\right)=\ldots \\
& \ldots=a_{1} a_{2} a_{3} a_{4}-a_{1} a_{2}-a_{1} a_{4}-a_{3} a_{4}+1,
\end{aligned}
$$

шестой:

$$
\begin{aligned}
v_{5}\left(a_{1}, \ldots, a_{5}\right) & =a_{1} a_{2} a_{3} a_{4} a_{5}-a_{1} a_{2} a_{3}-a_{1} a_{2} a_{5}- \\
& -a_{1} a_{4} a_{5}-a_{3} a_{4} a_{5}+a_{1}+a_{3}+a_{5}
\end{aligned}
$$

(проделайте эти вычисления самостоятельно!).

Внимательно посмотрев на эти выражения, можно заметить закономерность, которой они подчиняются. гурацию наподо-

$a_{1}, \ldots, a_{n}$. Теперь соединим между собой какие-нибудь пары соседних точек, с условием, что каждую точку можно соединить не более чем с одной соседней. Мы получим «код Морзе» из точек и тире - конфибие изображенной на рисунке 11.

$$
\begin{array}{ccccccc}
a_{1} & a_{2} & a_{3} & a_{4} & a_{5} & a_{6} & a_{7} \\
\circ & -0 & \circ & \circ & \circ & -\circ & \circ
\end{array}
$$

PUC. 11

Такой конфигурации сопоставим моном (т.е. одночлен): произведение переменных, которые стоят в точках (не соединенных с соседними), взятое со знаком «Плюс» или «минус» в зависимости от четности числа тире. Будем называть этот моном весом кода Морзе $c$ и обозначать через ж $(c)$. Так, конфигурации рисунка 11 будет отвечать выражение

$$
\omega(c)=(-1)^{2} a_{3} a_{4} a_{7}=a_{3} a_{4} a_{7}
$$

Пример 2. Все коды Морзе и соответствующие им мономы при $n$ от 1 до 5 представлены в таблице на рисунке 12.

Определение 2 (правило Эйлера). Континуантой $V_{n}\left(a_{1}, \ldots, a_{n}\right)$ порядка $n$ называется сумма одночленов, соответствующих всевозможным кодам Морзе на $n$ вершинах.

Замечание 2. Положим континуанту нулевого порядка (не зависящую от каких-либо аргументов) равной $V_{0}=1$. Более того, иногда бывает удобно считать, что $V_{n}=0$, если $n$ отрицательно.

Таблица (см. рис.12) позволяет нам вычислить континуанты порядков, не превосходящих 5. Мы видим, что у нас получаются в точности те же выражения, что и для $v_{n}\left(a_{1}, \ldots, a_{n}\right)$. Вскоре мы докажем это утверждение, но сначала установим еще некоторые свойства континуант. Начнем с рекуррентного соотношения. 


\begin{tabular}{|c|c|c|}
\hline$n=1$ & $\circ$ & $a_{1}$ \\
\hline$n=2$ & $\begin{array}{lr}0 & 0 \\
0 & -0\end{array}$ & $\begin{array}{c}a_{1} a_{2} \\
-1\end{array}$ \\
\hline$n=3$ & $\begin{array}{lcr}0 & 0 & 0 \\
0 & 0 & -0 \\
0 & -0 & 0\end{array}$ & $\begin{array}{c}a_{1} a_{2} a_{3} \\
-a_{1} \\
-a_{3}\end{array}$ \\
\hline$n=4$ & $\begin{array}{cccc}0 & 0 & 0 & 0 \\
0 & 0 & 0 & -0 \\
0 & 0 & -0 & 0 \\
0 & -0 & 0 & 0 \\
0 & -0 & 0 & -0\end{array}$ & $\begin{array}{c}a_{1} a_{2} a_{3} a_{4} \\
-a_{1} a_{2} \\
-a_{1} a_{4} \\
-a_{3} a_{4} \\
1\end{array}$ \\
\hline$n=5$ & $\begin{array}{ccccc} & 0 & 0 & 0 & 0 \\
0 & 0 & 0 & 0 & -0 \\
0 & 0 & 0 & 0 & 0 \\
0 & 0 & 0 & 0 & 0 \\
0 & -0 & 0 & 0 & 0 \\
0 & 0 & 0 & 0 & 0 \\
0 & -0 & 0 & 0 & 0 \\
0 & -0 & 0 & 0 & 0\end{array}$ & $\begin{array}{c}a_{1} a_{2} a_{3} a_{4} a_{5} \\
-a_{1} a_{2} a_{3} \\
-a_{1} a_{2} a_{5} \\
-a_{1} a_{4} a_{5} \\
-a_{3} a_{4} a_{5} \\
a_{1} \\
a_{3} \\
a_{5}\end{array}$ \\
\hline
\end{tabular}

Рис. 12. Коды Морзе при $n \leq 5$

Предложение 1. Континуанть удовлетворяют рекуррентному соотношению

$$
\begin{aligned}
V_{n}\left(a_{1}, \ldots, a_{n}\right)=a_{1} V_{n-1} & \left(a_{2}, \ldots, a_{n}\right)- \\
& -V_{n-2}\left(a_{3}, \ldots, a_{n}\right) . \quad(* *)
\end{aligned}
$$

Доказательство. Возьмем какой-нибудь код Морзе на $n$ вершинах. Посмотрим на первую вершину. Возможны два варианта. Либо ей отвечает точка, и тогда всякая конфигурация $с$ получается как код Морзе $c^{\prime}$ на вершинах 2, $\ldots, n$; при этом $w(c)=a_{1} w\left(c^{\prime}\right)$, поскольку в коде $c$ первая вершина учитывается, а в конфигурации $c^{\prime}$ нет. Либо же, напротив, первая вершина соединена со второй при помощи тире; в таком случае обозначим код Морзе на вершинах $3, \ldots, n$ через $c^{\prime \prime}$, а веса кодов $c$ и $c^{\prime \prime}$ отличаются знаком: $ш(c)=-w\left(c^{\prime \prime}\right)$.

Стало быть, все коды, начинающиеся с точки, дадут в континуанту $V_{n}\left(a_{1}, \ldots, a_{n}\right)$ вклад, равный $a_{1} V_{n-1}\left(a_{2}, \ldots, a_{n}\right)$, а все начинающиеся с тире - вклад, равный $-V_{n-2}\left(a_{3}, \ldots, a_{n}\right)$. Это и дает нам искомое соотношение $(* *)$.

Дальнейшие свойства континуант мы оставим в качестве упражнения для читателя.
Упражнение 5. Докажите следующие равенства:

(1) $V_{n}\left(a_{1}, \ldots, a_{n}\right)=V_{n}\left(a_{n}, \ldots, a_{1}\right)$;

(2) $V_{n}\left(a_{1}, \ldots, a_{n-1}, 0\right)=V_{n-1}\left(a_{1}, \ldots, a_{n-1}\right)$;

(3) $V_{n}\left(a_{1}, \ldots, a_{n}\right)=a_{n} V_{n-1}\left(a_{1}, \ldots, a_{n-1}\right)-$ $-V_{n-2}\left(a_{1}, \ldots, a_{n-2}\right)$.

Соотношение унимодулярности для континуант. Справедлива следующая теорема.

Теорема 1. Для любых $a_{1}, \ldots, a_{n+1}$ имеет место соотношение

$$
\begin{aligned}
& V_{n}\left(a_{1}, \ldots, a_{n}\right) V_{n}\left(a_{2}, \ldots, a_{n+1}\right)= \\
& \quad=V_{n-1}\left(a_{2}, \ldots, a_{n}\right) V_{n+1}\left(a_{1}, \ldots, a_{n+1}\right)+1 .
\end{aligned}
$$

Мы приведем два доказательства этой теоремы, алгебраическое и комбинаторное.

Алгебраическое доказательство. Будем доказывать эту теорему индукцией по $n$. База, при $n=1$, очевидна.

Запишем требуемое соотношение для $n$ и перепишем все члены, в которые входит $a_{n+1}$, с помощью равенства (3) из упражнения 5:

$$
\begin{aligned}
& V_{n+1}\left(a_{1}, \ldots, a_{n+1}\right)=a_{n+1} V_{n}\left(a_{1}, \ldots, a_{n}\right)- \\
&- V_{n-1}\left(a_{1}, \ldots, a_{n-1}\right) ; \\
& V_{n}\left(a_{2}, \ldots, a_{n+1}\right)=a_{n+1} V_{n-1}\left(a_{2}, \ldots, a_{n}\right)- \\
&-V_{n-2}\left(a_{2}, \ldots, a_{n-1}\right) .
\end{aligned}
$$

Тогда соотношение перепишется в виде

$$
\begin{aligned}
V_{n}\left(a_{1}, \ldots, a_{n}\right)\left(a_{n+1} V_{n-1}\left(a_{2}, \ldots, a_{n}\right)-\right. & \\
\left.-V_{n-2}\left(a_{2}, \ldots, a_{n-1}\right)\right)= & \\
=V_{n-1}\left(a_{2}, \ldots, a_{n}\right) & \left(a_{n+1} V_{n}\left(a_{1}, \ldots, a_{n}\right)-\right. \\
& \left.-V_{n-1}\left(a_{1}, \ldots, a_{n-1}\right)\right)+1 .
\end{aligned}
$$

В левой и правой частях нашего равенства встречается слагаемое $a_{n+1} V_{n-1}\left(a_{2}, \ldots\right.$ $\left.\ldots, a_{n}\right) V_{n}\left(a_{1}, \ldots, a_{n}\right)$; уничтожив его, получим равенство

$$
\begin{aligned}
& -V_{n}\left(a_{1}, \ldots, a_{n}\right) V_{n-2}\left(a_{2}, \ldots, a_{n-1}\right)= \\
& \quad=-V_{n-1}\left(a_{1}, \ldots, a_{n-1}\right) V_{n-1}\left(a_{2}, \ldots, a_{n}\right)+1,
\end{aligned}
$$

которое имеет место по предположению индукции. Теорема доказана.

Комбинаторное доказательство. Рассмотрим каждую из континуант в левой части соотношения как сумму мономов, отвечающим кодам Морзе на множествах $\{1, \ldots, n\}$ и $\{2, \ldots, n+1\}$ соответственно. Будем изображать эти коды Морзе на 
одном рисунке, только относящиеся к первому коду тире будем изображать пунктирными линиями, а ко второму - сплошными, как показано на рисунке 13. Таким

$0--0-0--0 \quad 0 \quad 0=0 \quad 0-0$

Рис. 13

образом, пунктирные тире могут стоять на всех возможных местах, кроме последнего, а сплошные - на всех, кроме первого. Тогда левая часть доказываемого соотношения равна сумме мономов, полученным по всевозможным парам кодов Морзе с такими условиями.

Сделаем то же для произведения континуант в правой части: каждое произведение мономов из $V_{n-1}\left(a_{2}, \ldots, a_{n}\right)$ и $V_{n+1}\left(a_{1}, \ldots, a_{n+1}\right)$ можно интерпретировать как пару кодов Морзе на множестве $\{1, \ldots, n+1\}$ - причем первый код (будем его обозначать пунктирными тире) не может иметь тире на первой и последней позициях, а на второй (обозначим его сплошными тире) никаких ограничений не накладывается.

Рассмотрим какую-либо конфигурацию $\left(c_{1}, c_{2}\right)$, где $c_{1}$ и $c_{2}-$ коды Морзе на $\{1, \ldots, n\}$ и $\{2, \ldots, n+1\}$ соответственно. Ей отвечает моном ж( $\left.c_{1}\right) w\left(c_{2}\right)$ из левой части равенства. Построим по ней новую конфигурацию $\left(c_{1}^{\prime}, c_{2}^{\prime}\right)$, где $c_{1}^{\prime}$ будет кодом Морзе на $\{1, \ldots, n+1\}$, а $c_{2}^{\prime}$ - кодом на $\{2, \ldots, n\}$.

Если между 1 и 2 в коде $c_{1}$ нет (пунктирного) тире, то $c_{1}$ можно рассматривать как код на вершинах $2, \ldots, n$. В то же время код из сплошных тире $c_{2}$ можно рассматривать как код на вершинах $1, \ldots, n+1$, у которого на первом месте нет тире. В таком случае положим $\left(c_{1}^{\prime}, c_{2}^{\prime}\right)=\left(c_{1}, c_{2}\right)$.

Пусть теперь вершины 1 и 2 соединены входящим в код $c_{1}$ пунктирным тире. Поскольку в код $c_{2}$ входят вершины, начинающиеся со 2-й, то между вершинами 1 и 2 нет сплошного тире. Построим по паре кодов $\left(c_{1}, c_{2}\right)$ пару кодов $\left(c_{1}^{\prime}, c_{2}^{\prime}\right)$ следующим образом: рассмотрим путь, выходящий из 1-й вершины и состоящий из пунктирных и сплошных тире. Ясно, что они могут только чередоваться. Заменим в нем каждое пунктирное тире на сплошное, а сплошное на пунктирное. Мы получим новую пару кодов $\left(c_{1}^{\prime}, c_{2}^{\prime}\right)$, у которой на первом месте стоит сплошное тире и нет пунктирного. Эта пара будет иметь тот же вес, что и $\left(c_{1}, c_{2}\right)$.

На рисунке 14 показана пара кодов, которые получаются такой операцией из

$$
0-0--0-0 \quad 0 \quad 0=-0 \quad 0-0
$$

Pис. 14

изображенных на рисунке 13. При этом пара $\left(c_{1}^{\prime}, c_{2}^{\prime}\right)$ будет «почти всегда» отвечать моному из правой части. Далее, эту процедуру «почти всегда» можно обратить: по «почти» каждой паре кодов $\left(c_{1}^{\prime}, c_{2}^{\prime}\right)$ можно единственным образом восстановить ту пару $\left(c_{1}, c_{2}\right)$, по которой она построена, причем веса этих пар будут равны.

Осталось разобраться с тем, что значат слова «почти всегда». Здесь нужно будет рассмотреть два случая: когда $n$ четно и когда оно нечетно.

Пусть $n$ четно. Тогда единственная конфигурация $\left(c_{1}, c_{2}\right)$, для которой описанная процедура не сработает (т.е. подходящего монома в правой части не найдется), будет иметь вид, как показано на рисунке 15. В ней Рис. 15 все вершины будут соединены чередующимися пунктирными и сплошными тире, причем и первое и последнее тире будут пунктирными (число вершин четно, значит, число тире между ними нечетно), а вес конфигурации $\left(c_{1}, c_{2}\right)$ будет равен $(-1)^{n-1}=-1$. Поэтому произведение континуант в левой части равенства будет на 1 меньше, чем произведение континуант в правой части.

Если же, напротив, число вершин нечетно, то у каждой конфигурации $\left(c_{1}, c_{2}\right)$ будет единственный образ $\left(c_{1}^{\prime}, c_{2}^{\prime}\right)$ - и найдется ровно одна конфигурация из правой части, у которой не будет прообраза. Она изображена на рисунке 16. В таком случае произведение континуант в

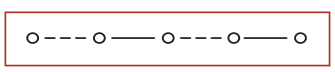

Рис. 16 правой части будет опять-таки на 1 больше, чем произведение континуант в левой части. 
Целочисленность фризов. Из результатов предыдущего подраздела немедленно получается следующий результат.

Теорема 2. Элемент $(n+1)-\check{u}$ строки фриза, над которым во второй строке стоят числа $a_{1}, \ldots, a_{n}$, равняется континуанте $V_{n}\left(a_{1}, \ldots, a_{n}\right)$.

Эта теорема сразу объясняет отмеченный нами выше «феномен целочисленности» - то, что все элементы фриза с целочисленной второй строкой $\left(a_{1}, a_{2}, \ldots\right)$ оказываются целыми числами, хотя, казалось бы, чтобы их вычислить, приходится совершать деление.

Следствие 1. Фриз, элементы второй строки которого целые положительные числа, является иелочисленным.

Действительно, континуанта - это многочлен от $\left(a_{1}, a_{2}, \ldots\right)$ с целыми коэффициентами.

\section{Восстановление второй строки по диа-} гонали. Итак, мы нашли способ вычислять диагональ фриза, зная вторую строку: это делается с помощью континуанты. Решим теперь обратную задачу: найдем (в обозначениях рисунка 10) вторую строку $a_{1}, \ldots, a_{n}$, зная диагональ $v_{2}, \ldots, v_{n-1}$.

Это непосредственно следует из рекуррентного соотношения для континуант. Следующая теорема - просто переформулировка предложения 1.

Теорема 3. Пусть $a_{1}, a_{2}, a_{3} \ldots$ - вторая строка фриза, $v_{1}=a_{1}, v_{2}, v_{3} \ldots-$ его диагональ. Тогда имеют место равенства

$$
a_{k}=\frac{v_{k-2}+v_{k}}{v_{k-1}} .
$$

(Напомним, что мы формально полагаем $v_{0}=1, v_{-1}=0$ - тогда эта формула применима при любых $k>0$ ).

Иногда эту теорему полезно формулировать в таком виде:

Следствие 2. Последовательность $v_{k}$ является решением разностного уравнения ( «равнения Хилла», или «дискретного уравнения Штурма-Лиувилля»)

$$
V_{k}=a_{k} V_{k-1}-V_{k-2},
$$

где $a_{1}, a_{2}, \ldots-$ коэффициенты, $V_{1}, V_{2}, \ldots-$ неизвестные.
Периодичность и скользящая симметрия. Рассмотрим фриз порядка $n$. Напомним, что такой фриз состоит из $n-1$ строки; последняя его строка состоит из единиц, а «следующая за последней», т.е. $n$-я, состоит из нулей. Таким образом, для его диагонали $1, v_{1}, \ldots, v_{n-1}$ имеют место равенства

$$
v_{n-2}=1 ; v_{n-1}=0 \text {. }
$$

Запишем последнее из рекуррентных соотношений. Оно имеет вид

$$
v_{n-1}=a_{n-1} v_{n-2}-v_{n-3} \text {, т.е. } v_{n-3}=a_{n-1} \text {. }
$$

Итак, мы получаем, что предпоследний ненулевой элемент диагонали $V_{n-3}$ равняется элементу $a_{n-1}$ из второй строки. Аналогично, следующий за ним в строке элемент равняется элементу $a_{n}$. Получается, что предпоследняя ненулевая строка фриза совпадает со второй, сдвинутой на $n / 2$ позиций, и фриз имеет такой вид, как показано на рисунке 17.

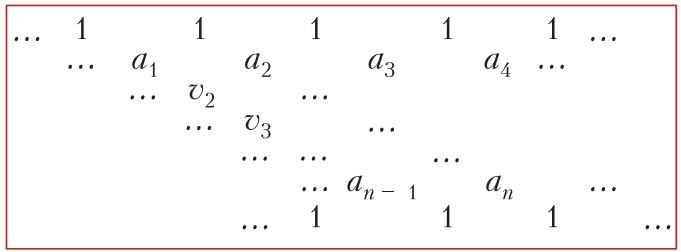

Pис. 17

А значит, из условия унимодулярности получается, что фриз можно строить не от второй строки вниз, а от предпоследней вверх, и результат будет тем же.

Следствие 3. Фриз порядка п обладает скользящей симметрией: его $k$-я строка, сдвинутая на n/2 позиций, совпадает с $(n-k)$-й строкой.

Следствие 4. Фриз порядка п является периодичным с периодом п.

В самом деле, сдвиг фриза на $n$ является результатом применения двух скользящих симметрий со сдвигом на $n / 2$.

\section{Целочисленные фризы}

Сущность целочисленного фриза. В этом разделе мы опишем все фризы, состоящие исключительно из целых элементов.

Будем называть сущностью (по-английски quiddity) фриза его вторую строку 
$\left(a_{1}, \ldots, a_{n}\right)$. Очевидно, она не может содержать двух единиц подряд - это противоречило бы унимодулярности. Однако оказывается, что хотя бы одна единица в этой строке обязательно должна быть.

Предложение 2. Вторая строка челочисленного фриза обязательно содержит 1.

Доказательство. Предположим, что это не так и что найдется фриз, все элементы во второй строке которого отличны от 1: $a_{k} \geq 2$. Тогда для элементов его диагонали (скажем, первой) имеет место неравенство

$$
v_{k}=a_{k} v_{k-1}-v_{k-2} \geq 2 v_{k-1}-v_{k-2},
$$

откуда

$$
v_{k}-v_{k-1} \geq v_{k-1}-v_{k-2} .
$$

Но это верно для всех $k$. Стало быть,

$$
\begin{array}{r}
v_{k}-v_{k-1} \geq v_{k-1}-v_{k-2} \geq \ldots \geq v_{2}-v_{1}= \\
=a_{2}-1 \geq 1,
\end{array}
$$

т.е. последовательность $v_{k}$ строго возрастающая. Это противоречит тому, что в какой-то момент она в силу замкнутости фриза должна принять значение 1 (и тем более 0$)$.

Следующая лемма позволит нам делать из данного целочисленного фриза порядка $n$ новый фриз порядка $n+1$.

Лемма. Пусть $\left(a_{1}, \ldots, a_{n}\right)-$ сущность челочисленного фриза порядка $n, u$ $1 \leq k \leq n$. Тогда:

(1) набор $\left(a_{1}, \ldots, a_{k-1}+1, a_{k}+1, a_{k+1}, \ldots\right.$ $\left.\ldots, a_{n}\right)$ является сущностью иелочисленного фриза порядка $n+1$;

(2) если 1, $v_{1}, v_{2}, \ldots, v_{n-1}-$ первая диагональ исходного фриза и $v_{1}=a_{1}$, то coomветствующая диагональ нового фриза имеет вид

$$
1, v_{1}, v_{2}, \ldots, v_{k-1}, v_{k-1}+v_{k}, v_{k}, \ldots, v_{n-2} .
$$

Доказательство. Начнем с доказательства пункта (2). Посмотрим, как будет устроена первая диагональ нового фриза: обозначим ее через $1, w_{1}, \ldots, w_{n-1}$.

Ясно, что при $i \leq k-2$ диагонали этих двух фризов совпадают: $w_{i}=v_{i}$. При этом на $(k-1)$-м месте нового фриза будет стоять

$$
w_{k-1}=\left(a_{k-1}+1\right) v_{k-2}-v_{k-3}=v_{k-1}+v_{k-2} .
$$

Далее,

$$
w_{k}=w_{k-1}-w_{k-2}=v_{k-1}+v_{k-2}=v_{k-1} .
$$

Следующий элемент диагонали будет равен

$$
\begin{aligned}
& w_{k+1}=\left(a_{k}+1\right) w_{k}-w_{k-1}= \\
& =\left(a_{k}+1\right) v_{k-1}-v_{k-1}-v_{k-2}= \\
& =a_{k} v_{k-1}-v_{k-2}=v_{k} .
\end{aligned}
$$

При всех $j>k+1$ будет иметь место равенство $w_{j}=v_{j-1}$. В частности, $(n-2)$-й и $(n-1)$-й члены этой последовательности будут равны 1 и 0 соответственно, т.е. фриз оборвется - а диагональ будет содержать $n$ положительных элементов. То же самое рассуждение применимо и к любой другой диагонали. Стало быть, в результате такой вставки получится целочисленный фриз на единицу большего порядка. Часть (1) также доказана.

Замечание 3. Эта конструкция очевидным образом является обратимой: если $\left(\ldots, b_{k-1}, 1, b_{k+1}, \ldots\right)$ - сущность целочисленного фриза, то и $\left(\ldots, b_{k-1}-1, b_{k+1}-1, \ldots\right)$ будет сущностью фриза на единицу меньшего порядка (обратите внимание, что в силу отсутствия двух единиц подряд оба числа $b_{k-1}-1$ и $b_{k+1}-1$ положительны).

Фризы и триангуляции. Рассмотрим выпуклый $n$-угольник с пронумерованными вершинами. Его триангуляцией будем называть его разбиение на треугольники с помощью диагоналей, не пересекающихся нигде, кроме вершин.

Замечание 4. Число триангуляций $n$ угольника называется $(n-2)-м$ числом Каталана (это одно из их многочисленных эквивалентных определений). Для него справедлива формула $C(n)=$ $=\frac{1}{n-1}\left(\begin{array}{c}2 n-4 \\ n-2\end{array}\right)$. О числах Каталана можно прочесть во многих книгах по комбинаторике; также о них неоднократно писал «Квант»- см., например, статью Мартина Гарднера в №7 за 1978 год.

Рассмотрим произвольную триангуляцию $n$-угольника. Ей можно сопоставить 
набор чисел $\left(c_{1}, \ldots, c_{n}\right)$, где $c_{i}-$ число треугольников, сходящихся в $n$-й вершине. Будем называть такой набор сущностью триангулящии. Триангуляция однозначно восстанавливается по своей сущности (почему?).

Теорема 4 (теорема Конвея-Кокстера). Набор $\left(c_{1}, \ldots, c_{n}\right)$, построенный по триангулящии п-угольника, является сущностью челочисленного фриза порядка п; это отображение устанавливает взаимно однозначное соответствие между триангуляциями п-угольника и целочисленными фризами п-го порядка.

Доказательство. Будем доказывать эту теорему по индукции по $n$. База $n=3$ очевидна: имеются лишь одна триангуляция треугольника и единственный фриз 3 -го порядка с сущностью $(1,1,1)$. (Читатель, которого это не убеждает, может начать с $n=4$.)

Рассмотрим некоторую триангуляцию $n$ угольника. Выберем в ней «ухо»- вершину, примыкающую к единственному треугольнику (в силу принципа Дирихле таких вершин не менее двух). Пусть эта вершина имеет номер $k$, а в вершинах $k-1$ и $k+1$ сходятся $b_{k-1}$ и $b_{k+1}$ треугольников соответственно. Таким образом, сущность этой триангуляции имеет вид $\left(\ldots, b_{k-1}, 1\right.$, $\left.b_{k+1}, \ldots\right)$. Выбросим треугольник, примыкающий к $k$-й вершине, из этой триангуляции, получим триангуляцию $(n-1)$-угольника, сущность которой равна $\left(\ldots, b_{k-1}-1\right.$, $\left.b_{k+1}-1, \ldots\right)$. Эта триангуляция, по предположению индукции, определяет фриз с той же сущностью. Но по лемме из предыдущего подраздела тогда и набор $\left(\ldots, b_{k-1}\right.$, $\left.1, b_{k+1}, \ldots\right)$ определяет целочисленный фриз.

Взаимная однозначность полученного соответствия следует из замечания 3: из сущности каждого фриза порядка $n$ можно удалить единицу, сведя дело к фризу на единицу меньшего порядка.

Восстановление фриза по триангуляции. Теорема Конвея-Кокстера утверждает, что каждый элемент второй строки фриза - это количество треугольников, сходящихся при соответствующей верши- не триангуляции. А как восстановить остальные элементы фриза? В чем их комбинаторный смысл?

Пусть дан целочисленный фриз, который мы будем обозначать в соответствии с рисунком 10: его вторая строка равна $\left(a_{1}, a_{2}, \ldots, a_{n}\right)$, а через $v_{0}=1, v_{1}, v_{2}, \ldots, v_{n-2}$ обозначены элементы диагонали, начинающейся в $a_{1}$, т.е. $v_{1}=a_{1}$. Удобно также считать, что $v_{-1}=0$.

Рассмотрим триангуляцию $n$-угольника, вершины которого пронумерованы числами от 1 до $n$, с сущностью $\left(a_{1}, \ldots, a_{n}\right)$. По ней можно восстановить $\left(v_{0}, v_{1}, v_{2}, \ldots\right)$ посредством следующего алгоритма:

- напишем в вершинах 1 и 2 числа 0 и 1 соответственно;

- далее расставим числовые отметки во всех остальных вершинах многоугольника по такому правилу: для каждого треугольника, в двух вершинах которого уже стоят числа $a$ и $b$, поставим в третьей его вершине число $a+b$.

Упражнение 6. Докажите, что во всех вершинах, соединенных с первой вершиной диагональю или стороной, будут написаны единицы.

Предложение 3. Полученная по этому алгоритму отметка в $i$-й вершине будет равна элементу $v_{i-2}$ диагонали фриза.

Доказательство. Данной триангуляции $n$-угольника сопоставим двойственный граф на $n-2$ вершинах: вершины его будут соответствовать треугольникам из триангуляции, причем две вершины будут соединены ребром, если у двух треугольников имеется общая сторона. Такой граф является деревом, валентности всех его вершин не превосходят трех, а висячие вершины отвечают «ушам» триангуляции (рис.18).

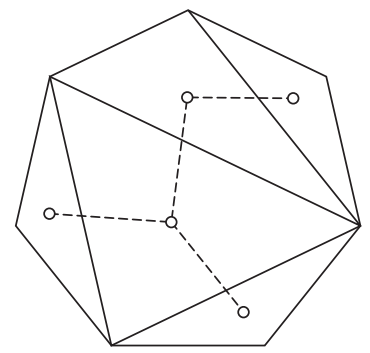

Рис. 18. Триангуляция и отвечающее ей дерево 
Далее, запись чисел в вершинах треугольников в соответствии с нашим алгоритмом можно рассматривать как обход вершин дерева, отвечающего триангуляции: на первом шаге алгоритма мы берем треугольник, содержащий вершины 1 и 2, и записываем число в его третьей вершине (для первого шага это число будет единицей). Сделав это, отметим отвечающую этому треугольнику вершину дерева как посещенную.

На каждом следующем шаге мы рассматриваем какую-то вершину, смежную с уже посещенными; ей отвечает треугольник, в двух вершинах которого уже стоят какието числа. Записав в третьей вершине треугольника их сумму, отметим как посещенную вершину дерева, которая отвечает этому треугольнику.

Докажем наше предложение по индукции по $n$. База при $n=3$ очевидна: мы получаем треугольник, в вершинах которого записаны 0,1 и 1 . Он отвечает единственному фризу порядка 3.

Индуктивный переход: пусть наше предложение доказано для всевозможных триангуляций $n$-угольника, а мы хотим доказать его для $(n+1)$-угольника. Посмотрим на последний шаг алгоритма; в его ходе мы обязаны посетить какую-то висячую вершину дерева триангуляции, т.е. добавить к триангуляции $n$-угольника «ухо». Пусть данный $n$-угольник имеет сущность $\left(a_{1}, \ldots, a_{n}\right)$, а «ухо» на последнем шаге приклеивается к стороне $(k-1, k)$. По предположению индукции, построенный в ре- зультате выполнения алгоритма набор отметок в вершинах $\left(v_{0}, \ldots, v_{n-1}\right)$ будет стоять на диагонали фриза с сущностью $\left(a_{1}, \ldots, a_{n}\right)$. Далее, на последнем шаге между вершинами $k-1$ и $k$ будет добавлена вершина с отметкой $v_{k-1}+v_{k}$. При этом триангуляция $(n+1)$-угольника будет иметь сущность $\left(\ldots, b_{k-1}+1,1, b_{k}+1, \ldots\right)-$ что согласуется с результатом леммы об элементах фриза. Предложение доказано.

\section{Упражнения}

7. Рассмотрим триангуляцию $n$-угольника, все диагонали которой образуют зигзаг (иными словами, все элементы сущности этой триангуляции не превосходят 3). Докажите, что все элементы соответствующего фриза будут числами Фибоначчи.

8. Пусть в триангуляции есть вершина, где сходятся не менее 4 треугольников. Докажите, что соответствующий фриз содержит число 4.

Замечание 5. Большинство результатов из этого раздела можно найти в двух работах Дж.Конвея и Д.Кокстера «Triangulated polygons and frieze patterns». Эти работы, опубликованные в двух последовательных номерах журнала The Mathematical Gazette (57(400, pp. 87-94; 401, pp. 175-183), 1973), посвященного вопросам математического образования, по форме представляют собой привычный ученикам математических кружков и классов листок с задачами. В первой статье ряд утверждений о фризах сформулирован в виде последовательности из примерно трех десятков задач, а во второй статье приводятся их решения или достаточно подробные указания.

\section{Вниманию наших читателей}

Начиная с 2017 года журнал “Квант» стал ежемесячным и в год выходит 12 номеров журнала.

Подписаться на наш журнал можно с любого номера в любом почтовом отделении. Наш подписной индекс в каталоге "Пресса России» - 90964.

Купить журнал "Квант» возможно в магазине "Математическая книга» издательства МЦНМО (адрес интернет-магазина: biblio.mccme.ru), а также в московских книжных магазинах "Библио-глобус», "Молодая гвардия», "Московский дом книги» и в редакции журнала. Архив вышедших номеров журнала "Квант» имеется на сайте http:/ / kvant.ras.ru 